\title{
Análisis
}

\section{Aplicaciones web para recuperación y análisis de bibliografía de PubMed}

\author{
Por Layla Michán-Aguirre, Roberto Calderón-Rojas, América Nitxin-Castañeda-Sortibrán \\ y Rosario Rodríguez-Arnáiz
}

\begin{abstract}
Resumen: Se analizan las aplicaciones disponibles en la Web para el uso de literatura especializada en ciencias biomédicas. Se identificaron, examinaron y describieron 28 utilidades académicas que realizan análisis automáticos. Son de uso amigable, mejoran la búsqueda, recuperación, manejo y meta-análisis (bibliometría, análisis de redes y minería de textos) de literatura de PubMed de manera eficiente, automatizada, actualizada y sistematizada, permiten procesar grandes cantidades de registros documentales simultáneamente y posibilitan elegir los más relevantes. Son iniciativas que están acordes con las necesidades y retos de la investigación biomédica de nuestro tiempo.

Palabras clave: PubMed, Web, Bibliometría, Análisis de redes, Minería de textos.

Title: Web applications for literature retrieval and analysis from PubMed

Abstract: This paper analyzes the Web applications to manage literature in Biomedical Sciences. We identified, examined and described 28 academic utilities that perform automated analysis. They are user-friendly, and altogether provide better search, retrieval, management and metaanalysis (bibliometrics, network analyses and text mining) of PubMed literature in an efficient, automated, updated and systematized manner that facilitates the simultaneous handling of large quantities of paper records, making it possible for the user to choose the most relevant. All of these initiatives address the needs and challenges of biomedical research in our time.
\end{abstract}

Keywords: PubMed, Web, Bibliometrics, Network analysis, Text mining.

Michán-Aguirre, Layla; Calderón-Rojas, Roberto; Nitxin-Castañeda-Sortibrán, América; Rodríguez-Arnaiz, Rosario. "Aplicaciones web para recuperación y análisis de literatura de PubMed". El profesional de la información, 2010, mayo-junio, v. 19, n. 3, pp. 285-291.

DOI: 10.3145/epi.2010.may.09

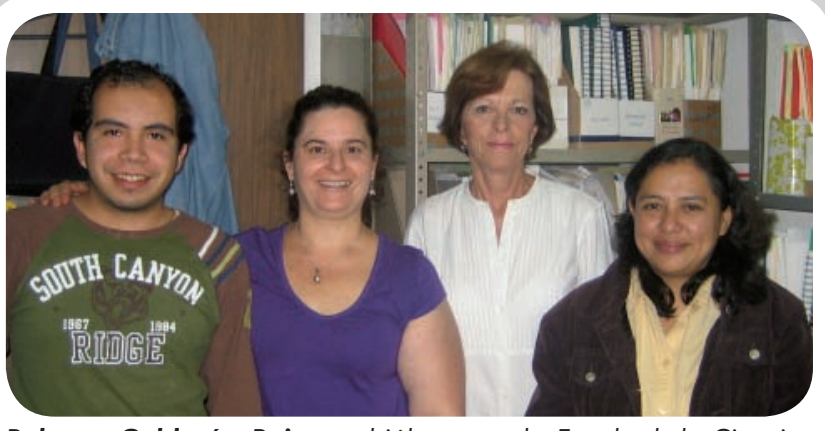

Roberto Calderón-Rojas es biólogo por la Facultad de Ciencias de la Universidad Nacional Autónoma de México (UNAM). Realizó su tesis de licenciatura sobre las aplicaciones disponibles en la Web para ciencias biomédicas. Imparte cursos y realiza publicaciones sobre e-investigación bibliográfica en ciencias biomédicas.

Layla Michán-Aguirre es investigadora y profesora en el Departamento de Biología Evolutiva de la Facultad de Ciencias de la Universidad Nacional Autónoma de México (UNAM). Estudia el impacto de la revolución digital en las ciencias biológicas durante las últimas décadas. Participa en proyectos de investigación sobre la estructura, el desarrollo y la dinámica de la biología aplicando bibliometría. Imparte cursos y diseña materiales para e-investigación bibliográfica en ciencias biomédicas.

Rosario Rodríguez-Arnáiz es investigadora y profesora en la Facultad de Ciencias de la UNAM. Es coordinadora del Laboratorio de Genética de la Facultad de Ciencias desde 1985. Dirige proyectos de investigación sobre la genotoxicidad de extractos de plantas naturales, en los ensayos Smart y Cometa usando como modelo a Drosophila melanogaster. Se interesa también en las aplicaciones de la Web para investigación en genética.

América Nitxin-Castañeda-Sortibrán es investigadora y profesora en la Facultad de Ciencias de la UNAM. Ha estudiado la genética de poblaciones en mariposas y actualmente estudia la genotoxicidad, así como el impacto de las tecnologías de la información y la comunicación en la enseñanza. Participa en proyectos de investigación sobre la genotoxicidad de extractos de plantas naturales, en los ensayos Smart y Cometa usando como modelo a Drosophila melanogaster.

\section{Introducción}

PUBMED DESTACA entre las bases de datos en línea especializadas en ciencias biomédicas por ser de consulta gratuita y tener la mayor cobertura mundial en ciencias de la vida y de la salud.
Admite búsquedas en una gran cantidad de campos y proporciona una serie de instrumentos que posibilitan al usuario personalizar y gestionar las búsquedas (Shultz, 2007; Falagas et al., 2008). Utiliza como vocabulario controlado la lista de encabezamientos Medical subject headings (MeSH) (Neveol et al., 2009) que contiene los términos controlados (Figueroa-Hernández et al., 2003) que representan materias de los documentos que -como es sabido- no necesariamente coin- 
ciden con las palabras empleadas en ellos.

Pubmed cuenta con cerca de 19 millones de registros, de los cuales 3 millones han sido publicados en los últimos 5 años. Medline, el componente principal de PubMed, recoge referencias bibliográficas de artículos publicados en 4.500 revistas biomédicas (medicina, oncología, enfermería, odontología, veterinaria, salud pública y ciencias preclínicas) desde 1964 (NIH, 2009).

El crecimiento exponencial de la bibliografía biomédica ha complicado la localización y recuperación de las publicaciones relevantes para una investigación.

En los últimos años la comunidad biomédica ha diseñado diversas herramientas con interfaces cómodas, desde sencillos complementos hasta sofisticadas aplicaciones para el análisis de registros bibliográficos (Hunter; Coen, 2006). Entre sus funciones se incluyen: seleccionar y clasificar los documentos, extraer información relevante, generar resúmenes y elaborar procesos complejos de análisis de miles de documentos simultáneamente mediante algoritmos.

La utilización de estas aplicaciones facilita y automatiza la recuperación de bibliografía, permitiendo obtener nuevo conocimiento y actualizarse, sin necesidad de una gran infraestructura ni asesoría personalizada de profesionales en bioinformática y/o ciencias de la computación (Jensen; Saric; Bork, 2006).

http://pubmed.gov

\section{"PubMed es la base de datos más usada en biomedicina debido a su cobertura y sobre todo a su gratuidad"}

\section{Material y método}

Se buscaron, caracterizaron, clasificaron y describieron las aplicaciones disponibles en la web que extraen y procesan registros de PubMed hasta diciembre de 2009, eligiéndose sólo aquellas que cubrieran los siguientes criterios: fines académicos, gratuitas, interfaz de usuario sencilla y que realizaran algún proceso de meta-análisis a partir de los registros:

- Bibliometría: análisis cuantitativo y estadístico de la producción de libros y documentos impresos.

- Análisis de redes: mapas diseñados mediante la teoría de redes de las conexiones más relevantes entre los nodos estudiados, que dan información sobre patrones y relaciones existentes (Perianes-Rodríguez; Olmeda-Gómez; De-MoyaAnegón, 2008).

- Minería de textos (text mining): descubrimiento de patrones y nuevos conocimientos (tendencias, desviaciones y asociaciones) a partir de la vinculación de un conjunto de textos mediante programas y algoritmos. (García-Marco, 2007; Sánchez-Cuadrado et al., 2007; Sánchez Jiménez; Gil-Urdiciain, 2007).

\section{Resultados}

Se identificaron 28 aplicaciones, que se clasificaron en los tres grupos de meta-análisis utilizados. Se muestra: nombre, url, editor y características principales.

\section{Bibliometría}

\section{HubMed}

http://www.hubmed.org/

Hubmed. Realiza búsquedas en Pubmed por fecha y relevancia (el número de veces que surge el término buscado en el título y en el resumen). A partir del resultado envía actualizaciones periódicas de la literatura publicada, agrupaciones y presentación gráfica de los artículos relacionados, ampliación de térmi- nos de consulta y la exportación directa de los metadatos de la referencia en varios formatos. Vincula las palabras clave a fuentes externas de información y clasificación manual (etiquetado) (Eaton, 2006).

\section{MedSum (The Medline summary tool) \\ http://webtools.mf.uni-lj.si/public/ medsum.html}

IBMI webtools. Realiza consultas sobre autores, revistas y temas de PubMed. Genera perfiles de autores, identifica las áreas de las revistas y crea gráficos de la evolución temporal o temática de la literatura, o sobre algún aspecto específico.

\section{Meva}

http://www.med-ai.com/meva/index. html

Institute for medical statistics and epidemiology. Procesa los resultados de una consulta en $P u b$ Med para examinar la bibliografía mediante una pregunta específica. La aplicación condensa la lista de registros bibliográficos en una gráfica de distribución de frecuencias, tablas de contingencia (tablas con los valores que toman las variables independientes y dependientes) y listas. Con base en el $\mathrm{MeSH}$, se enlistan los términos y códigos del mismo. Se pueden especificar los filtros o las frecuencias mínimas para restringir la búsqueda. Los resultados pueden obtenerse en html para su importación a cualquier base de datos para análisis posteriores.

\section{PubFocus \\ http://pubfocus.com/}

Pubfocus.com. Analiza al primer y último autor (que suelen ser el que realiza la mayor parte del trabajo y el jefe del grupo respectivamente) de los artículos de $P u b$ $\mathrm{Med}$. Procesa las revistas donde se ha publicado la mayor parte de los trabajos sobre el área, los tipos de documentos que hay y las revistas de mayor impacto. Es una muy bue- 


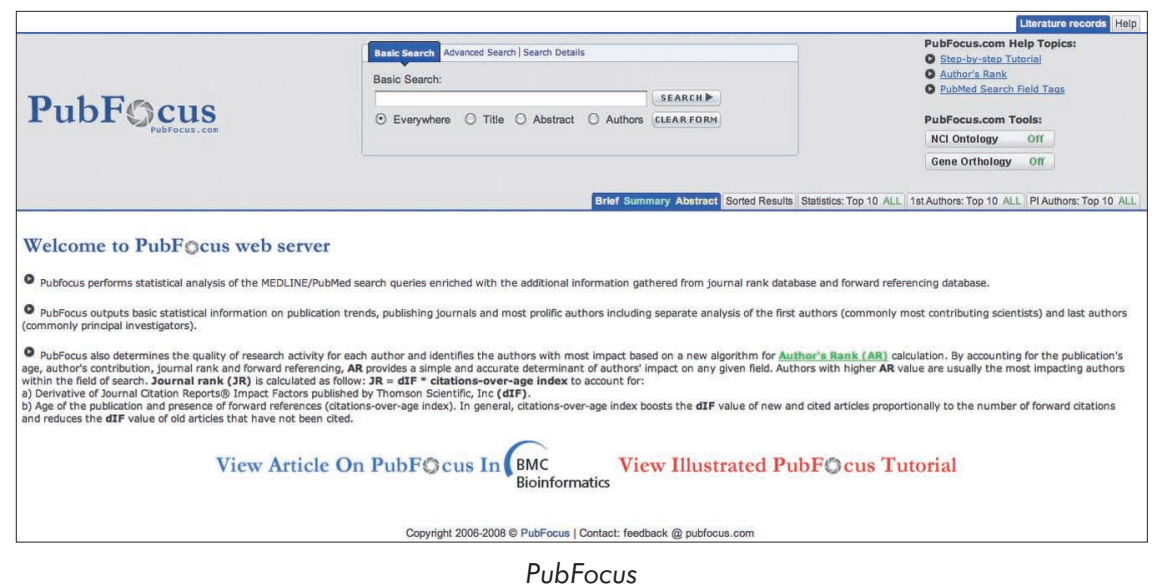

na alternativa para quien desea conocer la progresión de un área biomédica (Plikus; Zhang; Chuong, 2006).

\section{PubMed Faceoff}

http://www.postgenomic.com/faces/ index.php

Postgenomic.com. Proporciona información acerca del impacto de los artículos de PubMed a partir del análisis de sus citas, que se representan con un rostro humano. La edad de la cara se relaciona con la fecha de publicación del documento, siendo las caras más jóvenes las más recientes. Una sonrisa significa que el documento ha sido citado más veces de lo esperado (en función de su edad). Una sonrisa más amplia significa más citas. Una mueca significa que el texto ha sido citado pocas veces. Las cejas arqueadas se relacionan con el factor de impacto de la revista en la que se publicó el documento.

\section{PubReMiner \\ http://bioinfo.amc.uva.nl/human- genetics/pubreminer/}

Jan Koster (AMC) 2004, 2008. Obtiene frecuencias de una consulta en PubMed respecto a las revistas, autores, título, resumen, términos del $M e S H$, año y país de publicación. Todos los elementos se pueden agregar a la consulta y refinar los resultados para verlos de nuevo en $P u b$ Med. Permite una serie de opciones que funcionan como límitadores. $\mathrm{Al}$ realizar la búsqueda se despliegan unas tablas que organizan todos los artículos encontrados de acuerdo con su frecuencia (Douglas; Montelione; Gerstein, 2005).

\section{Twease}

http://twease.org/medline/app?co mponent=clearSettingsDirectLink Weill Medical College of Cornell University.

Busca en los resúmenes de $\mathrm{Me}$ dline e indiza todas las palabras que

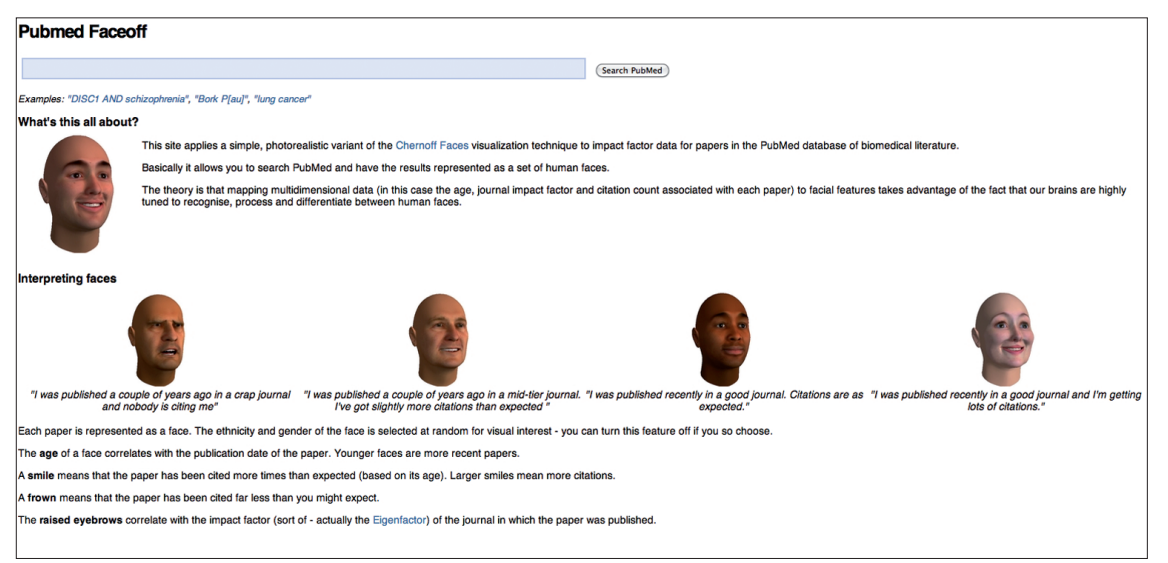

Pubmed Faceoff

se encuentran en ellos. Discierne entre palabras cortas muy parecidas de las abreviaturas comunes para un término. También muestra las estadísticas de la búsqueda (número de abreviaturas encontradas, tiempo que tardó en procesar la búsqueda, etc.). Al final muestra los artículos encontrados y los ordena según su relevancia, fecha de publicación o artículos relacionados.

\section{"Las aplicaciones de la web analizan miles de registros de PubMed clasifican documentos y extraen información relevante"}

\section{Análisis de redes}

\section{Ali Baba PubMed}

http://alibaba.informatik.hu-berlin. del

Universidad Humboldt de Berlín, Instituto de Informática. Muestra la conexión de los registros a partir de términos como células, fármacos, tejidos, enfermedades, reacciones, enzimas y compuestos -según el KEGG (Kyoto encyclopedia of genes and genomes)-, nutrientes, proteínas y genes de UniProt y especies de la NCBI Taxonomy. Una vez que los ha identificado muestra los vínculos de los artículos hallados como interacciones proteínaproteína, localización de proteínas, nutrientes y genes.

\section{Chilibot \\ http://www.chilibot.net/}

Encuentra relaciones entre proteínas, genes y palabras clave a partir de la búsqueda de dos términos, de una o dos listas de palabras en los resúmenes de PubMed. Restringe la búsqueda usando palabras clave y sinónimos para evitar ambigüedades y admite el uso de guías de regulación (inhibición, estimula- 


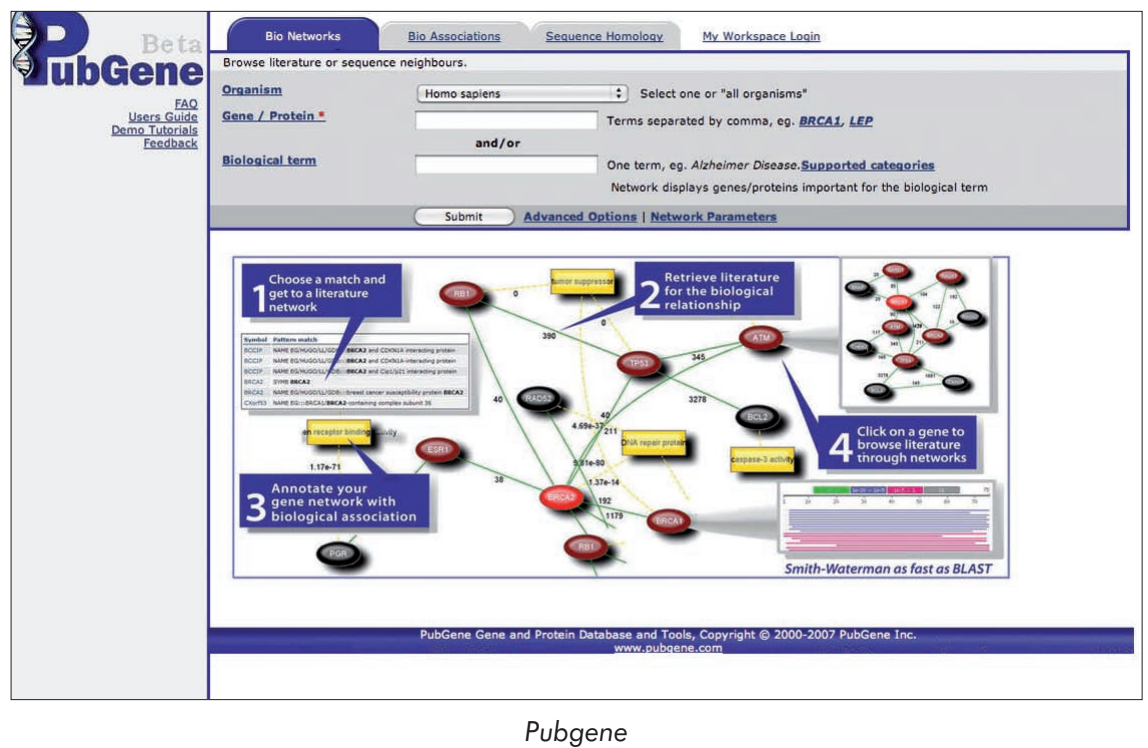

ción, aumento, reducción, etc.). El resultado gráfico muestra los nodos con colores. Dependiendo de los valores obtenidos existe la posibilidad de re-graficar los resultados, pues consigue generar subredes o eliminar nodos y relaciones.

\section{PubGene}

http://www.pubgene.org/tools/ Network/Subset.cgi

PubGene Inc. Encuentra las proteínas y los genes relacionados de los documentos. Muestra la cantidad de artículos correspondientes a cada nodo y sus relaciones.

PubNet (Publication network graph utility)

http://pubnet.gersteinlab.org/

"El uso de estas aplicaciones facilita y automatiza la recuperación de literatura biomédica"

Douglas, Shawn M.; Montelione, Gaetano T.; Gerstein, Mark, Gerstein Lab. Realiza búsquedas en PubMed y crea una red de la información introducida. Los nodos representan autores, artículos, genes de Gene Bank o proteínas de Swiss Prot. Las líneas representan las propiedades compartidas de la red, pueden ser co-autores o términos $\mathrm{MeSH}$, entre otros. La red se muestra en diversos formatos de archivo desde imágenes png hasta archivos pdf.

\section{Minería de textos}

\section{Arrowsmith}

http://arrowsmith.psych.uic.edu/ cgi-bin/arrowsmith_uic/start.cgi

The Arrowsmith Project, University of Illinois at Chicago, EBI. Busca conceptos comunes entre dos conjuntos de artículos. En la primera búsqueda se define la bibliografía $\mathrm{A}$, en la segunda la bibliografía C, y a partir de ambas el programa genera una lista con la bibliografía B que contiene un recopilado de frases y palabras claves que se comparten en los títulos de ambas (A y C). Se ordenan por relevancia y se pueden restringir a determinadas características semánticas, como regiones anatómicas, enfermedades o fármacos (Jensen; Saric; Bork, 2006).

\section{BioIE}

http://www.bioinf.manchester.ac.uk/ dbbrowser/bioie/

Universidad de Manchester. Extrae las oraciones informativas de la bibliografía biomédica sobre estructura, función, enfermedades, y compuestos terapéuticos, asi como localización, relaciones estructurales y funcionales entre las proteínas. Puede recuperar resúmenes de PubMed o bien se puede cargar texto propio.

\section{Biotext search engine}

http://biotext.berkeley.edu/

Universidad Berkeley de California. Indiza todas las tablas e imágenes de los artículos de consulta disponibles en PubMed Central. La colección se compone de más de 300 publicaciones periódicas. Los resultados pueden ser mostrados por relevancia del artículo o por fecha. Exhibe los resultados de las búsquedas en figuras, tablas, títulos, resumen, así como fragmentos y partes extraídas de los artículos (Hearst et al., 2007).

Bitola (Biomedical discovery support system)

http://ibmi.mf.uni-lj.si/bitola/?oe= bitola

IBMI Webtools. Descubre nuevas relaciones entre los conceptos biomédicos utilizando el conjunto de conceptos del MeSH y los genes humanos de HUGO.

\section{ClusterMed}

http://demos.vivisimo.com/vivisimo/ cgi-bin/query-meta? $v \% 3$ aproject $=$ clustermed \& \&v:frame $=$ form $\&$ frontpage $=1$

Vivisimo. Organiza los registros bibliográficos de PubMed en carpetas jerárquicas, lo que posibilita afinar los resultados más relevantes de forma rápida. Descubre los asuntos principales relacionados con la consulta realizada en forma de una lista cronológica. Agrupa los artículos similares con base en el autor, la fuente, fecha de publicación o términos $\mathrm{MeSH}$.

\section{ConceptLink}

http://project.cis.drexel.edu/concep tlinkl

Drexel. Interfaz visual para PubMed que crea imágenes de los conceptos médicos y sus relaciones. Guía al interesado en el diseño de las consultas mediante los 
mapas conceptuales que genera, identificando relaciones complejas. Proporciona funciones interactivas para explorar las relaciones entre los conceptos.

\section{EBIMed}

http://www.ebi.ac.uk/Rebholz-srv/ ebimed/

European Bioinformatics Institute. Realiza la co-ocurrencia de términos en los resúmenes de $P u b$ Med. Las frases extraídas y la terminología se utilizan para generar ontologías de las anotaciones, los fármacos y las especies utilizadas.

Fable (Fast automated biomedical literature extraction) http://fable.chop.edu/hgsid=null\& submitbutton $=$ View + browser $\&$ sub mithit=true

The Children's Hospital of Philadelphia. Obtiene los genes y las proteínas humanas, encuentra los artículos que citan un gen de interés (article finder), genera una lista de los genes asociados con una o más palabras clave (gene lister) y muestra un historial de la literatura disponible relacionada (LitTrack). El etiquetador de genes y el normalizador se utilizan para examinar todos los documentos en Medline y PubMed.

\section{GoPubMed}

http://www.gopubmed.org/web/ gopubmed

Gopubmed.org. Analiza la información de PubMed y presenta el sesgo de la producción, los autores que más publican y las relaciones entre ellos. Filtra los registros por los términos más comunes, autores o año de publicación. Los registros mostrados se pueden descargar, incluido el resumen cuándo está disponible en PubMed. Es una de las aplicaciones más elaboradas visualmente y fácil de usar por lo que es de las favoritas (Doms; Schoroeder, 2005).

\section{iHop (Information hiperlinked over proteins)}

http://www.ihop-net.org/UniPub/ iHOP/

Ihop-net.org. Proporciona los genes y proteínas que se asocian automáticamente con los resúmenes de PubMed. Es posible navegar por la bibliografía utilizando hipervínculos de los nombres de genes ocurrentes. Después de identificar un elemento de interés, el lector

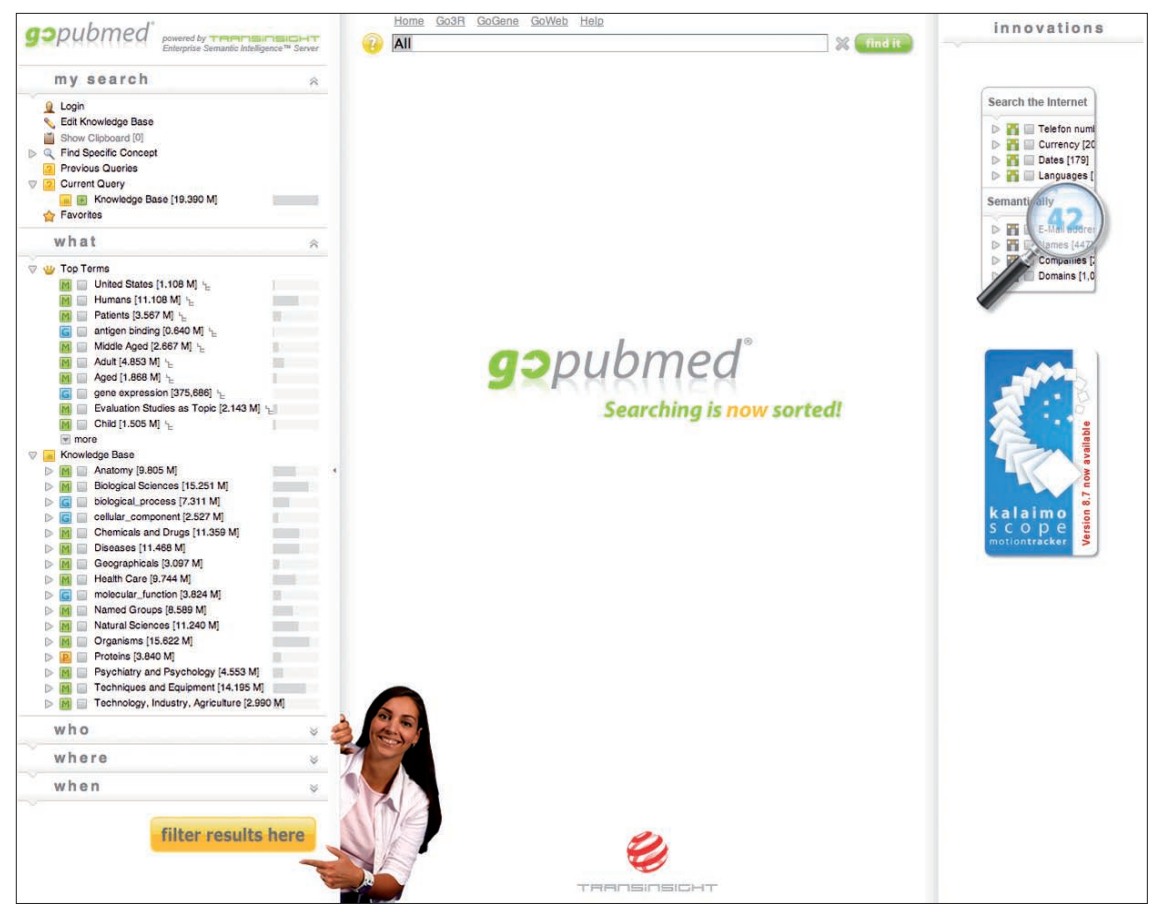

GoPubMed

puede ir a la página que contiene los nombres de los genes y las proteínas en los organismos a los que se refiere. También despliega una lista muy larga de posibles fragmentos de texto en los que se muestra una posible correlación entre genes y proteínas.

\section{Litlinker}

http://litlinker.ischool.washington. edu/

University of Washington. Identifica los vínculos posibles entre los términos biomédicos, hallando el conjunto de términos que se correlacionan con el término buscado. Para cada proceso de vinculación se emplea minería de textos para identificar conceptos de destino que se correlacionan con los conceptos de vinculación, que después se clasifican para mostrar las opciones de correlación conceptual entre los términos de búsqueda iniciales. El resultado es elegido por el usuario.

\section{LitMiner}

http://www.litminer.com

LitMiner. Anota las palabras clave en los resúmenes de los artículos y predice las relaciones entre los términos fundamentales de la bibliografía biomédica en cuatro categorías: genes, compuestos químicos, enfermedades y órganos. Posibilita realizar búsquedas en PubMed y en otras bases de datos para visualizar co-ocurrencias de los genes en la bibliografía.

\section{Medie \\ http://www-tsujii.is.s.u-tokyo.ac.jp/ mediel}

Tsujii Laboratory. Recupera correlaciones de resúmenes o enunciados mediante la especificación semántica de las correlaciones, por ejemplo, ¿qué activa p53? y ¿qué causa el cáncer de colon? Contiene casi 19 millones de artículos de PubMed.

\section{MedMiner}

http://biodatabase.org/index.php/ MedMiner 
Biodatabase.org. Filtra y organiza la información de los motores de búsqueda públicos, como GeneCards y PubMed.

\section{PubMatrix}

http://pubmatrix.grc.nia.nih.gov/

PubMatrix. Compara listas de términos de PubMed, hace posible la elaboración de tablas de relaciones entre palabras. Es útil para el análisis de conjuntos de datos combinatorios, como microarreglos de DNA, genómica, proteómica, o comparaciones múltiples. La base de datos PubMatrix es un archivo de búsquedas en muchos campos.

\section{PubMed-Ex}

http://bws.iis.sinica.edu.tw/Pub Med-EX

http://iisr.cse.yzu.edu.tw:8000/Pub Med-EX/

Tsai Richard T.; Lai Po T. y Huang Chi H., Universidad Chung $L i$, Taiwan. Extensión del navegador Firefox que marca los resultados de una consulta en PubMed con información adicional de minería de textos proveniente de los servicios del IISR (Institute of Information Science Research) y del IASL (Intelligent Agent Systems Lab). Establece en los registros secciones de categorización, como gen/enfermedad y el vínculo presente entre ellos. Puede ayudar a los investigadores a centrarse rápidamente en los términos clave y proporciona información adicional sobre ellos (Tsai et al., 2009).

\section{XplorMed}

http://www.ogic.ca/projects/xplor med/

Carolina Pérez-Iratxeta, Peer Bork, y Miguel A. Andrade. Muestra las principales asociaciones entre los términos relacionados en grupos de resúmenes. Se recomienda para los casos en que no se sabe exactamente qué se espera encontrar. En los resultados se sugerieren las palabras adicionales que pueden utilizarse para ampliar la consulta en PubMed.

\section{"En el meta-análisis de la bibliografía se incluyen: bibliometría, análisis de redes y minería de textos"}

\section{Conclusiones}

El crecimiento exponencial de la literatura biomédica actual y la ruptura de los límites disciplinarios, complica la localización y recuperación de las publicaciones relevantes para una investigación. La adopción generalizada de la búsqueda por título y palabras clave en Medline desde los años 70 dio lugar al primer gran cambio en la manera que los profesionales de la biomedicina recuperaron la literatura académica. Sin embargo, esto sólo representó el principio de la revolución informática, pronto aparecieron aplicaciones basadas en algoritmos que hacen posible recuperar, gestionar y procesar los registros de PubMed de forma rápida, dinámica y más sencilla. Muchas de ellas incluso aspiran a resolver tareas muy específicas que en la mayoría de los casos van más allá de la búsqueda bibliográfica, como el meta-análisis y algunos servicios propios de la web 2.0.

En este trabajo nos hemos limitado a hacer una breve descripción de los recursos. Algunas aplicaciones ya se han descrito con detalle en trabajos extensos, tal es el caso de Arrowsmith por Jensen, Saric y Bork en 2006, GoPubMed por Doms y Schoroeder en 2005, $\mathrm{Hu}$ bMed por Eaton en 2006, PubFocus por Plikus, Zhang y Chuong en 2006, PubMed-Ex por Tsai et al. en 2009, PubReminer por Douglas, Montelione y Gerstein en 2005 y Biotext por Hearst et al. en 2007. Esos estudios han sido realizados por los diseñadores de las aplicaciones y consisten en revisiones sistemáticas con enfoques en las áreas disciplinares de los propios creado- res. En ningún caso se proporciona una valoración, aunque sería interesante en un futuro, realizar una evaluación detallada de la fiablilidad, ventajas y desventajas de cada una de ellas.

Es recomendable que los usuarios tengan prudencia al interpretar los datos, pues hay que tener presente que proceden de sistemas automatizados. Para evitar errores hay que establecer una estrategia de búsqueda adecuada, manejar apropiadamente las fuentes de información y poner en práctica habilidades para analizar críticamente los resultados.

Las 28 aplicaciones para $P u b$ Med descritas en este documento están disponibles en la Web, son de uso gratuito y constituyen formas alternativas de búsqueda. Todas ellas son amigables, hacen más sencillo el proceso de plantearse preguntas y encontrar las respuestas correspondientes. Convierten la investigación bibliográfica en ciencias biomédicas en un proceso automatizado, eficiente, inmediato, actualizado y organizado. Esto facilita el manejo de grandes cantidades de registros documentales simultáneamente, permite elegir la información más relevante, manejar los registros seleccionados y examinarlos con base en métodos innovadores acorde con las necesidades y retos de nuestro tiempo, pero sobre todo, favorecen que sea un proceso más sencillo y agradable.

\section{Agradecimientos}

Dgapa, UNAM Proyecto Papime, PE 201509.

\section{Referencias}

Doms, Andreas; Schroeder, Michael. "GoPubMed: exploring PubMed with the Gene Ontology". Nucleic acids research, 2005, v. 33, suppl. 2, W783-786.

http://nar.oxfordjournals.org/cgi/content/full/33/ suppl_2/W783

Douglas, Shawn M.; Montelione, Gaetano T.; Gerstein, Mark. "PubNet: a flexible system for visualizing literature derived networks". Genome biology, 2005, v. 6, n. 9, R80.

http://www.ncbi.nlm.nih.gov/pubmed/16168087 
Eaton, Alfred D. "HubMed: a web-based biomedical literature search interface". Nucleic ac ids research, 2006, v. 34, suppl. 2, W745-747. http://nar.oxfordjournals.org/cgi/content/ abstract/34/suppl_2/W745

Falagas, Matthew E.; Pitsouni, Eleni I.; Malietzis, George A.; Pappas, Georgios. "Comparison of PubMed, Scopus, Web of Science, and Google Scholar: strengths and weaknesses". The Faseb journal 2008, v. 22, n. 2, pp. 338-342. http://www.fasebj.org/cgi/content/abstract/ 22/2/338

Figueroa-Hernández, José-Luis; Lorenzana, Marte; Lares-Assef, Ismael; MoncadaHernández, Sandra. "Fuentes de información III: búsquedas bibliográficas especializadas". En: Programa de estudios teórico-práctico de farmacología. Fac de Medicina, Depto de Farmacología, UNAM, 2003

García-Marco, Francisco-Javier. "Ontologías y organización del conocimiento: retos y opor tunidades para el profesional de la información". El profesional de la informacion, 2007, nov.-dic., v. 16 , n. 6 , pp. 541-550.

http://www.elprofesionaldelainformacion.com/ contenidos/2007/noviembre/01.pdf

Giglia, Elena. "Medline/PubMed revisited: new, semantic tools to explore the biomedical literature". European journal of physical and rehabilitation medicine, 2009, v. 45, n. 2, 293-297.

Hearst, Marti A.; Divoli, Anna; Guturu, Harendra; Ksikes, Alex; Nakov, Preslav; Wooldridge, Michael A.; Ye, Jerry. "BioText search engine: beyond abstract search". Bioinformatics, 2007, v. 23, n. 16, pp. 2196-21977. http://bioinformatics.oxfordjournals.org/cgi/ content/full/23/16/2196? view $=$ long \& pmid= 17545178
Hunter, Lawrence; Cohen, Bretonnel K. "Biomedical language processing: what's beyond PubMed?" Molecular cell, 2006, v. 21, n. 5, pp 589-594.

http://linkinghub.elsevier.com/retrieve/pii/ S1097276506001146

Jensen, Juhl Lars; Saric, Jasmin; Bork, Peer "Literature mining for the biologist: from information retrieval to biological discovery". Nature reviews genetics, 2006, v. 7, n. 2, pp. 119-129.

http://jean.aguilar.googlepages.com/Literature miningforthebiologist.pdf

National Institutes of Health. "PubMed Home" U.S. National Library of Medicine, 2009.

http://www.ncbi.nlm.nih.gov/sites/entrez?db= pubmed

Neveol, Aurelie; Mork, James G.; Aronson, Alan R. "Comment on 'MeSH-up: effective $\mathrm{MeSH}$ text classification for improved document retrieval". Bioinformatics, 2009, v. 25, n. 20, pp 2770-2771.

Perianes-Rodríguez, Antonio; Olmeda-Gómez, Carlos; De-Moya-Anegón, Félix. "Introducción al análisis de redes". El profesional de la informacion, 2008, nov.-dic., v. 17, n. 6, pp. 664-669.

Plikus, Maksim; Zhang, Zina; Chuong, Cheng-Ming. "PubFocus: semantic Medline/ PubMed citations analytics through integration of controlled biomedical dictionaries and ranking algorithm". BMC Bioinformatics, 2006, v. 7, n. 1 , pp. 424

http://www.biomedcentral.com/1471-2105/7/424

Shultz, Mary. "Comparing test searches in PubMed and Google Scholar". Journal of the Medical Library Association: JMLA, 2007, v. 95, n. 4, pp. 442-445.
http://www.ncbi.nlm.nih.gov/pmc/articles/PMC 2000776/

Sánchez-Cuadrado, Sonia; Morato-Lara, Jorge; Palacios-Madrid, Vicente; Llorens-Morillo, Juan; Moreiro-González, José-Antonio. "De repente, ¿todos hablamos de ontologías?" El profesional de la información, 2007, nov.-dic., v. 16 , n. 6, pp. 562-568.

http://www.elprofesionaldelainformacion.com/ contenidos/2007/noviembre/03.pdf

Sánchez-Jiménez, Rodrigo; Gil-Urdiciain, Blanca. "Lenguajes documentales y ontologías". El profesional de la información, 2007, nov.-dic., v. 16, n. 6 , pp. 551-560.

http://www.elprofesionaldelainformacion.com/ contenidos/2007/noviembre/02.pdf

Tsai, Richard T.; Dai, Hong-Jie; Lai, Po-Ting; Huang, Chi-Hsin. "PubMed-Ex: a web browser extension to enhance PubMed search with text mining features". Bioinformatics, 2009, v. 25, n. 22, pp. 3031-3032.

http://www.ncbi.nlm.nih.gov/pubmed/19654114

Layla Michán-Aguirre, Roberto Calderón-Rojas, América NitxinCastañeda-Sortíbrán y Rosario Rodríguez-Arnaiz. Facultad de Ciencias, UNAM

laylamichan@ciencias.unam.mx artaban421@gmail.com nitxin@ciencias.unam.mx rosario.rodriguez@ciencias.unam. $m x$

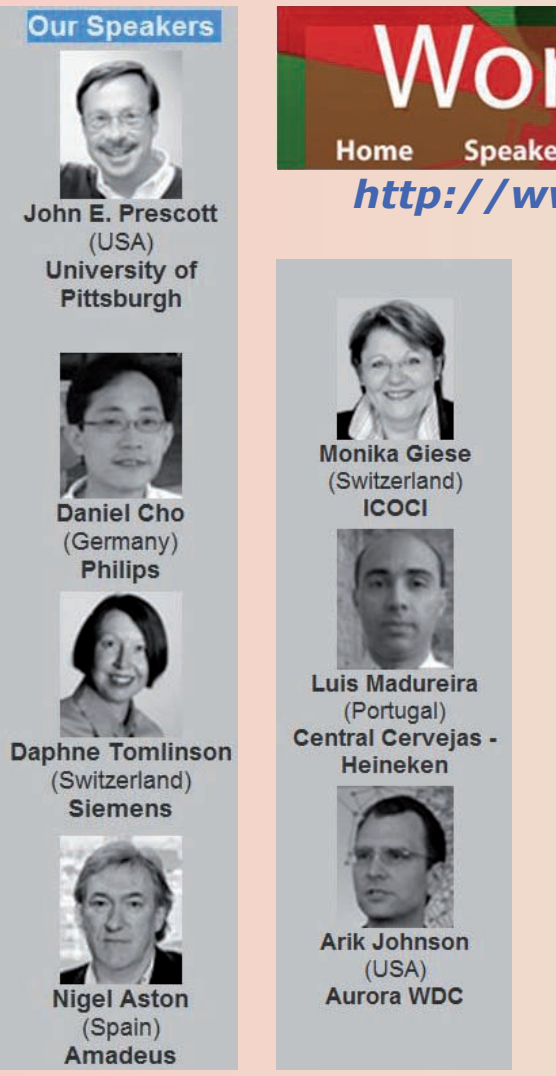

\section{Barcelona, 12 de julio de 2010}

Workshop práctico de primera clase, dirigido a analistas de información y profesionales de inteligencia competitiva (CI) para aprender y compartir ideas y experiencias.

Se analizarán casos reales de empresas de varios países, presentados por sus protagonistas, que proporcionarán métodos de recogida y análisis de datos, realización de informes y su presentación a la alta dirección.

Se trata de una oportunidad única de trabajar de forma interactiva con profesionales de inteligencia competitiva de otras compañías, aprendiendo las mejores prácticas.

Habrá presentaciones breves, actividades en pequeños grupos y discusiones plenarias. 